Article

\title{
Asymptotic approximation of central binomial coefficients with rigorous error bounds
}

\section{Richard P. Brent}

Australian National University Canberra, ACT 2600, Australia.; central@rpbrent.com

Academic Editor: Wei Gao

Received: 15 September 2021; Accepted: 23 October 2021; Published: 18 December 2021.

Abstract: We show that a well-known asymptotic series for the logarithm of the central binomial coefficient is strictly enveloping in the sense of Pólya and Szegö, so the error incurred in truncating the series is of the same sign as the next term, and is bounded in magnitude by that term. We consider closely related asymptotic series for Binet's function, for $\ln \Gamma\left(z+\frac{1}{2}\right)$, and for the Riemann-Siegel theta function, and make some historical remarks.

Keywords: Asymptotic series; Binet function; Binomial coefficient; Central binomial coefficient; Gamma function; Riemann-Siegel theta function; Stirling's approximation; strictly enveloping series.

MSC: 05A10; 11B65; 33B15; 41A60.

\section{Introduction}

$\mathbf{L}$

et $z \in \mathbb{C}$ and assume that $\Re z>0$. It is well-known that

$$
\ln \Gamma(z)=\left(z-\frac{1}{2}\right) \ln z-z+\frac{1}{2} \ln (2 \pi)+J(z),
$$

where $J(z)$ can be written as

$$
J(z)=\frac{1}{\pi} \int_{0}^{\infty} \frac{z}{\eta^{2}+z^{2}} \ln \left(\frac{1}{1-e^{-2 \pi \eta}}\right) d \eta .
$$

The analytic function $J(z)$ is known as Binet's function and has several equivalent expressions; see for example, Henrici [1, (8.5-7)].

Binet's function has an asymptotic expansion

$$
J(z) \sim \frac{\beta_{0}}{z}-\frac{\beta_{1}}{z^{3}}+\frac{\beta_{2}}{z^{5}}-\cdots,
$$

or more precisely, for non-negative integers $k$,

$$
J(z)=\sum_{j=0}^{k-1}(-1)^{j} \frac{\beta_{j}}{z^{2 j+1}}+r_{k}(z),
$$

where

$$
\beta_{k}=\frac{1}{\pi} \int_{0}^{\infty} \eta^{2 k} \ln \left(\frac{1}{1-e^{-2 \pi \eta}}\right) d \eta
$$

and

$$
r_{k}(z)=\frac{(-1)^{k}}{\pi z^{2 k-1}} \int_{0}^{\infty} \frac{\eta^{2 k}}{z^{2}+\eta^{2}} \ln \left(\frac{1}{1-e^{-2 \pi \eta}}\right) d \eta .
$$

It may be shown that

$$
\beta_{k}=\frac{2(2 k) !}{(2 \pi)^{2 k+2}} \zeta(2 k+2)=\frac{(-1)^{k}}{(2 k+1)(2 k+2)} B_{2 k+2} ，
$$


where $B_{2 k+2}$ is a Bernoulli number $\left(B_{2}=1 / 6, B_{4}=-1 / 30\right.$, etc.). Proofs of these results are given in Henrici's book [1, §11.1]. ${ }^{1}$ As far as possible, we have followed Henrici's notation.

Substituting (4) into (1) gives an asymptotic expansion for $\ln \Gamma(z)$ that is usually named after James Stirling, although some credit is due to Abraham de Moivre. For the history and early references, see Dutka [2]. It is interesting to note that de Moivre started (about 1721) by trying to approximate the central binomial coefficient $\left(\begin{array}{c}2 n \\ n\end{array}\right)$, not the factorial (or Gamma) function - see Dutka [2, pg. 227].

It is easy to see from (5) and (6) that

$$
r_{k}(z)=\theta_{k}(z)(-1)^{k} \frac{\beta_{k}}{z^{2 k+1}}
$$

where

$$
\theta_{k}(z)=\int_{0}^{\infty} \frac{z^{2} \eta^{2 k}}{z^{2}+\eta^{2}} \ln \left(\frac{1}{1-e^{-2 \pi \eta}}\right) d \eta / \int_{0}^{\infty} \eta^{2 k} \ln \left(\frac{1}{1-e^{-2 \pi \eta}}\right) d \eta
$$

Suppose now that $z$ is real and positive. Since $z^{2} /\left(z^{2}+\eta^{2}\right) \in(0,1)$ and the logarithmic factors in (9) are positive for all $\eta \in(0, \infty)$, we see that

$$
\theta_{k}(z) \in(0,1)
$$

Thus, the remainder $r_{k}(z)$ given by (8) has the same sign as the next term $(-1)^{k} \beta_{k} / z^{2 k+1}$ in the asymptotic series, and is smaller in absolute value. In the terminology used by Pólya and Szegö [3, Ch. 4], the asymptotic series for $\ln \Gamma(z)$ strictly envelops ${ }^{2}$ the function $\ln \Gamma(z){ }^{3}$

$\$ 2$ shows that we can deduce a strictly enveloping asymptotic series for $\ln \left(\Gamma(2 z+1) / \Gamma(z+1)^{2}\right)$ or equivalently, if $z=n$ is a positive integer, for the logarithm of the central binomial coefficient $\left(\begin{array}{c}2 n \\ n\end{array}\right)$. The series itself is well known, but we have not found the enveloping property or the resulting error bound mentioned in the literature. Henrici was aware of it, since in his book [1, §11.2, Problem 6] he gave the special case $k=3$ as an exercise, along with a hint for the solution. Hence, we do not claim any particular originality. Our purpose is primarily to make some useful asymptotic series and their associated error bounds readily accessible. Related results and additional references may be found, for example, in [4-6].

In $\$ 2$ we consider the central binomial coefficient and its generalisation to a complex argument. Then, in $\S 3$, we consider some closely related asymptotic series that we can prove to be strictly enveloping. In $\S 4$ we make some remarks on asymptotic series that are not enveloping. An Appendix gives numerical values of the coefficients appearing in three of the asymptotic series.

Finally, we remark that it is possible to give asymptotic series related to $\Gamma\left(z+\frac{1}{2}\right) / \Gamma(z)$ and $\left(\begin{array}{c}2 n \\ n\end{array}\right)$, but in general these series do not alternate in sign. See, for example, [7], [8], [9, ex. 9.60 and pg. 602], [10], and [11].

\section{Asymptotic series for central binomial coefficients}

Define

$$
\begin{aligned}
\widetilde{\Gamma}(z) & :=\frac{\Gamma(2 z+1)}{\Gamma(z+1)^{2}}, \\
\widetilde{J}(z) & :=J(2 z)-2 J(z), \\
\widetilde{r}_{k}(z) & :=r_{k}(2 z)-2 r_{k}(z),
\end{aligned}
$$

and

$$
\widetilde{\beta}_{k}:=\left(2-2^{-2 k-1}\right) \beta_{k}=(-1)^{k} \frac{\left(1-4^{-k-1}\right)}{(k+1)(2 k+1)} B_{2 k+2} .
$$

As noted above, the central binomial coefficient $\left(\begin{array}{c}2 n \\ n\end{array}\right)$ is simply $\widetilde{\Gamma}(n)$.

Using elementary properties of the Gamma function, we have

$$
\widetilde{\Gamma}(z)=\frac{2}{z} \frac{\Gamma(2 z)}{\Gamma(z)^{2}}
$$

1 There is an error in Henrici's equation (11.1-13): $2^{-2 \pi \eta}$ should be replaced by $e^{-2 \pi \eta}$.

We refer to the English translation. In the original it is "in engerem Sinne umhüllen".

When testing the enveloping property, we only consider the nonzero terms in the asymptotic series. See [3, Problem 142, footnote 1]. 
Thus, from (1) and the same equation with $z \mapsto 2 z$, we have

$$
\ln \widetilde{\Gamma}(z)=\ln \left(\frac{4^{z}}{\sqrt{\pi z}}\right)+\widetilde{J}(z)
$$

Also, from (4) and the definition of $\widetilde{J}(z)$, we have an asymptotic series for $\widetilde{J}(z)$, namely:

$$
\widetilde{J}(z)=-\sum_{j=0}^{k-1}(-1)^{j} \frac{\widetilde{\beta}_{j}}{z^{2 j+1}}+\widetilde{r}_{k}(z) .
$$

Since $\left(\begin{array}{c}2 n \\ n\end{array}\right)=\widetilde{\Gamma}(n)$, equations (13)-(14) give an asymptotic series for $\ln \left(\begin{array}{c}2 n \\ n\end{array}\right)$. Lemma 1 shows that the remainder $\widetilde{r}_{k}(z)$ can be expressed as an integral analogous to the integral (6) for $r_{k}(z)$.

Lemma 1. For $z \in \mathbb{C}, \Re z>0$, and $k$ a non-negative integer,

$$
\begin{gathered}
\widetilde{\beta}_{k}=-\frac{1}{\pi} \int_{0}^{\infty} \eta^{2 k} \ln \tanh (\pi \eta) d \eta, \\
\widetilde{r}_{k}(z)=\frac{(-1)^{k}}{\pi z^{2 k-1}} \int_{0}^{\infty} \frac{\eta^{2 k}}{z^{2}+\eta^{2}} \ln \tanh (\pi \eta) d \eta,
\end{gathered}
$$

and

$$
\widetilde{J}(z)=\widetilde{r}_{0}(z)
$$

Proof. Making the change of variables $z \mapsto 2 z$ and $\eta \mapsto 2 \eta$ in (6), we obtain

$$
r_{k}(2 z)=\frac{(-1)^{k}}{\pi z^{2 k-1}} \int_{0}^{\infty} \frac{\eta^{2 k}}{z^{2}+\eta^{2}} \ln \left(\frac{1}{1-e^{-4 \pi \eta}}\right) d \eta
$$

Now

$$
\ln \left(\frac{1}{1-e^{-4 \pi \eta}}\right)-2 \ln \left(\frac{1}{1-e^{-2 \pi \eta}}\right)=\ln \left(\frac{1-e^{-2 \pi \eta}}{1+e^{-2 \pi \eta}}\right)=\ln \tanh (\pi \eta),
$$

so (16)-(17) follow from the definitions of $\widetilde{r}_{k}(z)$ and $\widetilde{J}(z)$. The proof of (15) is similar.

Corollary 1 gives a result analogous to Equations (8)-(9).

Corollary 1. For $z \in \mathbb{C}, \Re z>0$, and $k$ a non-negative integer,

$$
\widetilde{r}_{k}(z)=\widetilde{\theta}_{k}(z)(-1)^{k+1} \frac{\widetilde{\beta}_{k}}{z^{2 k+1}},
$$

where

$$
\widetilde{\theta}_{k}(z)=\int_{0}^{\infty} \frac{z^{2} \eta^{2 k}}{z^{2}+\eta^{2}} \ln \tanh (\pi \eta) d \eta / \int_{0}^{\infty} \eta^{2 k} \ln \tanh (\pi \eta) d \eta .
$$

Proof. This is straightforward from Equations (15)-(16) of Lemma 1.

Corollary 2 gives a result analogous to the bound (10).

Corollary 2. If $z$ is real and positive, then $\widetilde{\theta}_{k}(z) \in(0,1)$.

Proof. We write (19) as

$$
\widetilde{\theta}_{k}(z)=\frac{\int_{0}^{\infty} \frac{z^{2} \eta^{2 k}}{z^{2}+\eta^{2}} \ln \operatorname{coth}(\pi \eta) d \eta}{\int_{0}^{\infty} \eta^{2 k} \ln \operatorname{coth}(\pi \eta) d \eta} .
$$

Observe that $\operatorname{coth}(y)=\cosh (y) / \sinh (y)>1$ for $y \in(0, \infty)$, so $\ln \operatorname{coth}(y)>0$ for $y=\pi \eta>0$. Since $z^{2} /\left(z^{2}+\eta^{2}\right) \in(0,1)$ for real positive $z$ and $\eta$, the result follows. 
Corollary 3. If $z$ is real and positive, then the asymptotic series (14) for $\widetilde{J}(z)$ is strictly enveloping.

Proof. This is immediate from Corollary 2.

Remark 1. We may compare Corollary 2 with (the proof of) Lemma 2.7 of [12]. The latter, after allowing for different notation, gives the bound

$$
\frac{-1}{4^{k+1}-1}<\widetilde{\theta}_{k}(z)<\frac{4^{k+1}}{4^{k+1}-1} .
$$

This is clearly weaker than the bound of Corollary 2, and not sufficient to prove Corollary 3.

\section{Some related asymptotic series}

Lemma 2. If $z \in \mathbb{C}, \Re z>0$, then

$$
\widetilde{J}(z)=\ln \left(\frac{\Gamma\left(z+\frac{1}{2}\right)}{z^{1 / 2} \Gamma(z)}\right) .
$$

Proof. This follows from Equations (12)-(13) and the duplication formula $\Gamma(z) \Gamma\left(z+\frac{1}{2}\right)=2^{1-2 z} \pi^{1 / 2} \Gamma(2 z)$.

From Lemma 2 and (14) we immediately obtain an asymptotic expansion

$$
\ln \left(\frac{\Gamma\left(z+\frac{1}{2}\right)}{\Gamma(z)}\right) \sim \frac{\ln z}{2}+\sum_{j \geq 0}(-1)^{j+1} \frac{\widetilde{\beta}_{j}}{z^{2 j+1}}
$$

which is strictly enveloping if $z$ is real and positive.

Define

$$
\widehat{\beta}_{j}=\widetilde{\beta}_{j}-\beta_{j}=\left(1-2^{-2 j-1}\right) \beta_{j} .
$$

Using the asymptotic expansion for $\ln \Gamma(z)$ given by Equations (1) and (3), we see from (21) that $\ln \Gamma\left(z+\frac{1}{2}\right)$ has an asymptotic expansion

$$
\ln \Gamma\left(z+\frac{1}{2}\right) \sim z \ln z-z+\frac{1}{2} \ln (2 \pi)+\sum_{j \geq 0}(-1)^{j+1} \frac{\widehat{\beta}_{j}}{z^{2 j+1}} .
$$

In fact, the expansion (23) was already obtained by Gauss [13, Eqn. [59] of Art. 29] in 1812. However, Gauss did not explicitly bound the truncation error. Writing (23) as

$$
\ln \Gamma\left(z+\frac{1}{2}\right)=z \ln z-z+\frac{1}{2} \ln (2 \pi)+\sum_{j=0}^{k-1}(-1)^{j+1} \frac{\widehat{\beta}_{j}}{z^{2 j+1}}+\widehat{r}_{k}(z),
$$

we have an unsurprising result for the truncation error $\widehat{r}_{k}(z)$ : the error is of the same sign as the first neglected term $(-1)^{k+1} \widehat{\beta}_{k} / z^{2 k+1}$, and is bounded in magnitude by this term. This is shown in Lemma 3 and Corollaries $4-5$ below.

Lemma 3. For $z \in \mathbb{C}, \Re z>0$, and $k$ a non-negative integer,

$$
\widehat{\beta}_{k}=\frac{1}{\pi} \int_{0}^{\infty} \eta^{2 k} \ln \left(1+e^{-2 \pi \eta}\right) d \eta
$$

and

$$
\widehat{r}_{k}(z)=\frac{(-1)^{k+1}}{\pi z^{2 k-1}} \int_{0}^{\infty} \frac{\eta^{2 k}}{z^{2}+\eta^{2}} \ln \left(1+e^{-2 \pi \eta}\right) d \eta .
$$

Proof. This is similar to the proof of Lemma 1.

Corollary 4. For $z \in \mathbb{C}, \Re z>0$, and $k$ a non-negative integer,

$$
\widehat{r}_{k}(z)=\widehat{\theta}_{k}(z)(-1)^{k+1} \frac{\widehat{\beta}_{k}}{z^{2 k+1}},
$$


where

$$
\widehat{\theta}_{k}(z)=\int_{0}^{\infty} \frac{z^{2} \eta^{2 k}}{z^{2}+\eta^{2}} \ln \left(1+e^{-2 \pi \eta}\right) d \eta / \int_{0}^{\infty} \eta^{2 k} \ln \left(1+e^{-2 \pi \eta}\right) d \eta .
$$

Proof. This is a straightforward consequence of Lemma 3.

Corollary 5. If $z$ is real and positive, then the asymptotic expansion for $\ln \Gamma\left(z+\frac{1}{2}\right)$ given in (24) is strictly enveloping.

Proof. From (28) we have $\widehat{\theta}_{k}(z) \in(0,1)$.

Remark 2. If we make the change of variables $z \mapsto n+\frac{1}{2}$ in (23), and assume that $n$ is a positive integer, we obtain an asymptotic series for $n !$ in negative powers of $\left(n+\frac{1}{2}\right)$ :

$$
\ln n ! \sim\left(n+\frac{1}{2}\right) \ln \left(n+\frac{1}{2}\right)-\left(n+\frac{1}{2}\right)+\frac{1}{2} \ln (2 \pi)+\sum_{j \geq 0}(-1)^{j+1} \frac{\widehat{\beta}_{j}}{\left(n+\frac{1}{2}\right)^{2 j+1}} .
$$

In fact, (29) was stated (without proof) by de Moivre [14,15] as early as 1730, see Dutka [2, (5), pg. 233].

\section{Non-enveloping asymptotic series}

Lest the reader has gained the impression that all "naturally occurring" asymptotic series are enveloping (for real positive arguments), we give two classes of examples to show that this is not the case. In fact, enveloping series are the exception, not the rule. Our first class of examples is given by the following Lemma.

Lemma 4. Let $x \in(0,+\infty)$ and $f(x):=J(x)+\exp (-b x)$ for some constant $b \in(0,2 \pi)$. Then $f(x)$ has an asymptotic series

$$
f(x) \sim \sum_{j=0}^{\infty}(-1)^{j} \frac{\beta_{j}}{x^{2 j+1}} .
$$

However, the series (30) does not envelop $f$.

Proof. For all $k \geq 0, \exp (-b x)=O\left(x^{-2 k-1}\right)$ as $x \rightarrow+\infty$. Thus, it follows from (4) that $f(x)$ has the claimed asymptotic series (in fact the same series as the Binet function $J$.) This proves the first claim.

To prove the final claim, suppose, by way of contradiction, that the series (30) envelops $f$. For each integer $k>0$, define $x_{k}:=k / \pi$. From (7), the $\beta_{k}$ grow like $(2 k) ! /(2 \pi)^{2 k}$, and from Stirling's approximation we see that

$$
\beta_{k} / x_{k}^{2 k+1}=O\left(\exp \left(-2 \pi x_{k}\right)\right) \text { as } k \rightarrow \infty .
$$

Since the same series envelops both $f$ and $J$, (31) implies that

$$
\left|f\left(x_{k}\right)-J\left(x_{k}\right)\right|=O\left(\exp \left(-2 \pi x_{k}\right)\right) \text { as } k \rightarrow \infty .
$$

Since $\exp (-2 \pi x)=o(\exp (-b x))$, it follows that, for sufficiently large $k$,

$$
\left|f\left(x_{k}\right)-J\left(x_{k}\right)\right|<\exp \left(-b x_{k}\right) .
$$

This contradicts the definition of $f$, so the assumption that the series (30) envelops $f$ must be false.

Remark 3. Lemma 4 can be generalised. For example, the conclusion holds if $f(x)=J(x)+g(x)$, where $g(x)=O\left(x^{-k}\right)$ for all positive integers $k$, but $g(x) \neq O(\exp (-2 \pi x))$. Also, we can replace the function $J(x)$ by a different function that has an enveloping asymptotic series whose terms grow at the same rate as those of $J(x)$.

Our second class of examples involves asymptotic expansions where all (or all but a finite number) of the terms are of the same sign (assuming a positive real argument $x$ ). Such series can not be strictly enveloping [3, Ch. 4]. As examples, we mention the Bessel function $I_{0}(x)$ (see Olver and Maximon [16, \$10.40.1]), the product of two Bessel functions $I_{0}(x) K_{0}(x)$ (see [16, §10.40.6] and [17, Lemma 3.1]), and the Riemann-Siegel 
theta function (see $[18, \S 6.5]$ ). In all these examples the terms have constant sign, so the remainder changes monotonically as the number of terms increases with the argument $x$ fixed. Eventually the remainder changes sign and starts increasing in absolute value. Often the point where the remainder changes sign is close to where the terms are smallest in absolute value, but this is not always true - see for example [19, $\S \S 4-5]$.

\section{Concluding remarks}

We have considered three different but related asymptotic series that can all be proved to be strictly enveloping. Our proofs depend on the fact that the three relevant functions $-\ln \left(1-e^{-2 \pi \eta}\right), \ln \operatorname{coth}(\pi \eta)$, and $\ln \left(1+e^{-2 \pi \eta}\right)$ are positive for all $\eta \in(0, \infty)$. We remark that these three functions are linearly dependent, since

$$
\operatorname{coth}(\pi \eta)=\frac{1+e^{-2 \pi \eta}}{1-e^{-2 \pi \eta}}
$$

It follows that the sequences $\left(\beta_{k}\right)_{k \geq 0},\left(\widetilde{\beta}_{k}\right)_{k \geq 0}$ and $\left(\widehat{\beta}_{k}\right)_{k \geq 0}$ are linearly dependent. In fact, $\widetilde{\beta}_{k}=\beta_{k}+\widehat{\beta}_{k}$ for all $k \geq 0$. A table of numerical values is given in the Appendix.

Acknowledgments: We thank an anonymous referee for simplifying the proof of Lemma 4 and for noting that the domain of validity of (2) is the right half-plane $\Re z>0$ (although the Binet function $J(x)$ may be continued into the left half-plane by analytic continuation, see [1, Thm. $8.5 \mathrm{a}])$.

Conflicts of Interest: "The author declares no conflict of interest".

\section{References}

[1] Henrici, P. (1991). Applied and Computational Complex Analysis, 2. Wiley Classics Library, New York.

[2] Dutka, J. (1991). The early history of the factorial function. Archive for History of Exact Sciences, 43, 225-249.

[3] Pólya, G. \& and Szegö, G. (1978). Problems and Theorems in Analysis I. Springer Classics in Mathematics (D. Aeppli, translator). Springer, Berlin.

[4] Nemes, G. (2013). Generalization of Binet's Gamma function formulas. Integral Transforms and Special Functions, 24, 597-606.

[5] Olver, F. W. J. (1974). Asymptotics and Special Functions. Academic Press, New York.

[6] Askey, R. A. \& Roy, R. (2021).Gamma Function. Ch. 5 in NIST Digital Library of Mathematical Functions. http:/ / dlmf.nist. gov/.

[7] Dyson, F. J., Frankel, N. E., \& Glasser, M. L. (2013). Lehmer's interesting series. The American Mathematical Monthly, 120, 116-130.

[8] Elezović, N. (2014). Asymptotic expansions of central binomial coefficients and Catalan numbers. Journal of Integer Sequences, 17, article 14.2.1.

[9] Graham, R. L., Knuth, D. E., \& Patashnik, O. (1994). Concrete Mathematics, 2nd edition, Addison-Wesley, New York.

[10] Kessler, D. \& Schiff, J. (2006). The asymptotics of factorials, binomial coefficients and Catalan numbers. Preprint. http:/ / u.math.biu.ac.il/ schiff/Papers/prepap3.pdf.

[11] Lehmer, D. H. (1985). Interesting series involving the central binomial coefficient. The American Mathematical Monthly, 92, 449-457.

[12] Brent, R. P., Osborn, J. H., \& Smith, W. D. (2016). Probabilistic lower bounds on maximal determinants of binary matrices. The Australasian Journal of Combinatorics, 66, 350-364.

[13] Gauss, C. F. (1813). Disquisitiones generales circa seriem infinitam etc. Reprinted in Carl Friedrich Gauss Werke, Bd. 3, Göttingen, 1876, 123-162. https:/ /archive.org/details/werkecarlf03gausrich.

[14] de Moivre, A. (1730). Miscellaneis Analyticus Supplementum. London.

[15] de Moivre A. (1756). The Doctrine of Chances: A Method of Calculating the Probabilities of Events in Play, 3rd edition, London.

[16] Olver, F. W. J. \& Maximon, L. C. (2021). Bessel Functions. Ch. 10 in NIST Digital Library of Mathematical Functions, http://dlmf.nist.gov/.

[17] Brent, R. P. \& Johansson, F. (2015). A bound for the error term in the Brent-McMillan algorithm. Mathematics of Computation, 84, 2351-2359.

[18] Edwards, H. M. (1974). Riemann's Zeta Function. Academic Press, New York. Reprinted by Dover Publications, 2001.

[19] Brent, R. P. (2019). On asymptotic approximations to the log-Gamma and Riemann-Siegel theta functions. Journal of the Australian Mathematical Society, 107, 319-337.

[20] Sloane, N. J. (2019). The Best Writing on Mathematics 2019: The On-Line Encyclopedia of Integer Sequences (pp. 90-119). Princeton University Press. 


\section{Appendix: Numerical values of the coefficients}

The table below gives the exact values of the coefficients $\beta_{k}, \widetilde{\beta}_{k}$ and $\widehat{\beta}_{k}$ for $0 \leq k \leq 6$. The values have been computed from equations (7), (11) and (22). We recall from the discussion above that the coefficients occur in the asymptotic expansions

$$
\begin{aligned}
\ln \Gamma(z) & \sim\left(z-\frac{1}{2}\right) \ln z-z+\frac{1}{2} \ln (2 \pi)+\frac{\beta_{0}}{z}-\frac{\beta_{1}}{z^{3}}+\frac{\beta_{2}}{z^{5}}-\cdots, \\
\ln \left(\begin{array}{c}
2 n \\
n
\end{array}\right) & \sim \ln \left(\frac{4^{n}}{\sqrt{\pi n}}\right)-\frac{\widetilde{\beta}_{0}}{n}+\frac{\widetilde{\beta}_{1}}{n^{3}}-\frac{\widetilde{\beta}_{2}}{n^{5}}+\cdots, \text { and } \\
\ln \Gamma\left(z+\frac{1}{2}\right) & \sim z \ln z-z+\frac{1}{2} \ln (2 \pi)-\frac{\widehat{\beta}_{0}}{z}+\frac{\widehat{\beta}_{1}}{z^{3}}-\frac{\widehat{\beta}_{2}}{z^{5}}+\cdots,
\end{aligned}
$$

the $\widehat{\beta}_{k}$ also occurring in de Moivre's series (29) and, with a different sign pattern, in a series related to the Riemann-Siegel theta function [19, eqn. (2)]: $2 \vartheta(t) \sim t \ln (t / 2 \pi e)-\pi / 4+\widehat{\beta}_{0} / t+\widehat{\beta}_{1} / t^{3}+\cdots$. In all but the Riemann-Siegel theta function case the asymptotic series are strictly enveloping, so the error incurred in truncating the series can be bounded by the first term omitted, provided that $z \in(0, \infty)$ is real and that $n$ is a positive integer. For error bounds if $z$ is complex, we refer to [19, §\$2-3].

\begin{tabular}{c|c|c|c}
$k$ & $\beta_{k}$ & $\widetilde{\beta}_{k}$ & $\widehat{\beta}_{k}$ \\
\hline 0 & $1 / 12$ & $1 / 8$ & $1 / 24$ \\
\hline 1 & $1 / 360$ & $1 / 192$ & $7 / 2880$ \\
\hline 2 & $1 / 1260$ & $1 / 640$ & $31 / 40320$ \\
\hline 3 & $1 / 1680$ & $17 / 14336$ & $127 / 215040$ \\
\hline 4 & $1 / 1188$ & $31 / 18432$ & $511 / 608256$ \\
\hline 5 & $691 / 360360$ & $691 / 180224$ & $1414477 / 738017280$ \\
\hline 6 & $1 / 156$ & $5461 / 425984$ & $8191 / 1277952$
\end{tabular}

Remark 4. We note that the sequence $\left((-1)^{k} \beta_{k}\right)_{k \geq 0}$ is in the Online Encyclopedia of Integer Sequences (OEIS) [20]. The (signed) numerators are sequence A046968, and the denominators are sequence A046969. The sequence $\left(\widehat{\beta}_{k} / 2\right)_{k \geq 0}$ is also in the OEIS: the numerators are sequence A036282, and the denominators are sequence A114721. We have added the sequence $\left((-1)^{k} \widetilde{\beta}_{k}\right)_{k \geq 0}$ to the OEIS. The (signed) numerators are sequence A275994, and the denominators are sequence A275995. Other relevant sequences are A143503 and A061549. 